\title{
The importance of culture-antibiogram and evaluation of the empirical antibiotic treatment in peritonitis due to perforated appendicitis acquired intra-abdominal infection.
}

\author{
Hakan Sezgin Sayiner $^{*}$, Sadık Akgün², Hasan Öğünç Apaydın³, Mustafa Göksu" ${ }^{4}$ Nail Aksoy ${ }^{5}$, İlkay \\ Akgün6, Musa Abes ${ }^{3}$ \\ ${ }^{1}$ Department of Clinical Infectious Diseases and Medical Microbiology, Faculty of Medicine, Adiyaman University, \\ Training and Research Hospital, Adiyaman, Turkey \\ ${ }^{2}$ Department of Medical Microbiology, Faculty of Medicine, Adiyaman University, Training and Research Hospital, \\ Adiyaman, Turkey \\ ${ }^{3}$ Department of Pediatric Surgery, Faculty of Medicine, Adiyaman University, Training and Research Hospital, \\ Adiyaman, Turkey \\ ${ }^{4}$ Department of General Surgery, Faculty of Medicine, Adiyaman University, Training and Research Hospital, \\ Adiyaman, Turkey \\ ${ }^{5}$ Department of Pediatric Surgery, Faculty of Medicine, Kars University, Kars, Turkey \\ ${ }^{6}$ Intensive Care Unit, Adıyaman University, Adiyaman, Turkey
}

\begin{abstract}
Prudent use of antibiotics gains more importance as increased antibiotic resistance along with a gradual recession in newly developed antibiotics is seen. It is aimed to show the importance of cultureantibiogram for detecting causative bacteria and antibiotic resistance profile for prudent antibiotic usage and empirical antibiotic coverage in pediatric and adult cases with peritonitis secondary to perforated appendicitis. This study includes a total of 58 patients who underwent surgery for perforated appendicitis and secondary peritonitis. Cultures-antibiogram obtained by suctioning of the peritoneal fluid or swabbing during the surgery of the patients having proliferation was evaluated. Out of total 35 pediatric cases, $E$. coli in 27 cases, $E$. coli and $P$. aeruginosa in 5 cases. In the 32 cases with $E$. coli cultured, antibiotic resistance was seen for Ampisilin-sulbactam (SAM) in 13 (36.1\%) cases, ceftriaxone in $12(33.3 \%)$ cases and Extended Spectrum Beta Lactamase (ESBL) in $10(31.2 \%)$ cases. Out of total 23 adult cases, $E$. coli was cultured in 18 cases. Of the 18 cases with $E$. coli cultured, antibiotic resistance was seen for SAM in $10(43.4 \%)$ cases, ceftriaxone in $9(39.1 \%)$ cases and ESBL in $6(33.3 \%)$ cases. It is believe that the evaluation of peritoneal culture results obtained during surgery will lead to a decrease in mortality and morbidity rates.
\end{abstract}

Keywords: Antibiotic resistance, Community infections, Perforated appendicitis, Secondary peritonitis.

Accepted on January 25, 2018

\section{Introduction}

Since the discovery of the first antibiotic, penicillin, incidentally in 1928 by Alexander Fleming, there has been a continuous race against the bacteria. Though humanity initially believed that the war against the bacteria could easily be won, bacteria have evolved continuously against antibiotics by developing resistance mechanisms. As the number of newly discovered or developed antibiotics decrease, the new strategy of humanity shifted to the prudent use of antibiotics. The main step in paradigm shift is initiation of treatment based on culture antibiogram results, when empirical antibiotic treatment is required, culture antibiogram results from similar patient group can help to guide treatment. One of these scenarios is peritoneal infection as a result of peritoneal inflammation.

If peritonitis occurs due to perforation, inflammation or ischemia of luminal abdominal organs like appendix, colon or recum, it is described as secondary peritonitis [1].

Secondary Peritonitis (SP) is an acute peritoneal infection due to an intraabdominal pathology, loss of gastrointestinal or genitourinary tract integrity and microbial contamination. SP is 
polymicrobial with a predominance of gram negative bacilli with an enteric flora origin. For a successful treatment, supportive therapy, surgical source control and antimicrobial therapy to eliminate microbial contamination are essential [2].

In this study, to investigate whether there is difference in causative bacteria and choose of empirical antibiotic therapy between pediatric and adult population in patients with a perforated appendicitis diagnosed either by ultrasonography or computed tomography, culture of sampled fluids, culture antibiogram results and choose of empirical antibiotic treatment were retrospectively reviewed.

\section{Materials and Methods}

A total of 58 patients' test results were included in the study that were accepted to Adiyaman University, Pediatric Surgery and General Surgery Service, and in whom perforated appendicitis was detected on abdominal USG or tomography, between December 2013 and September 2015, retrospectively. Of these patients, 35 were children $($ age $<18)$ and 23 were adults (age $\geq 18$ ). Empiric antibiotic was initiated to patients at the primary healthcare center where they applied after their complaints had been started. Symptoms of perforation were detected after 1 to $4 \mathrm{~d}$. Otherwise, no history of hospitalization and therapy were present within the last 3 months in patients. Of the pediatric patients, $12(342 \%)$ were female and 23 $(65.7 \%)$ were male, the mean age was 11.2 (youngest 4 , oldest $17) ; 10(43.4 \%)$ were women and $13(56.5 \%)$ were male in adults, and the mean age was 44.4 (youngest 18, oldest 74 ). Patients having proliferation their cultures obtained by suctioning of the peritoneal fluid or swabbing during the surgery, and their results of culture-antibiogram were evaluated.

In microbiology laboratory, accepted samples were routinely cultured in Petri dishes containing commercial Sheep Blood Agar (SBA) and Eosin-Methylene-Blue agar (EMB). The dishes were incubated in bacteriological incubators at $37^{\circ} \mathrm{C}$ for 18 to $24 \mathrm{~h}$. Microbiological evaluation was performed on positively evaluated plagues at the end of this period (18 to 24 h). Certain conventional tests (catalase, coagulase, oxidase and others) and stained microscopic examination (distinction of gram positive or gram negative by gram staining) were performed contributively to identification on microorganisms. Suitable colonies were obtained and processed in complete automatized culture-antibiogram sensitivity device (BD Phoenix 100; Becton, Dickinson and Co., 21152 USA) by using favorable kits (BD Phoenix NMIC/ID-99 UNMIC/ID-83, PMIC/ID-70, SMIC/ID-11) in accordance with the recommendations of manufacturer. Sensitivity results of identification and antibiotic were reported approximately after 8 to $12 \mathrm{~h}$.

\section{Results}

Culture and antibiogram results of 58 patients with peritonitis due to perforated appendicitis in Pediatric Surgery and General
Surgery Clinics were examined. Of these, 35 were children $($ age $<18)$ and 23 were adults (age $\geq 18$ ).

Among 35 pediatric patients, only E. coli was detected in 27 (77\%). E. coli and $P$. aeruginosa was detected in $5(14 \%)$ and only $P$. aeruginosa, Klebsiella oxytoca and Enterococcus faecalis were detected in $1(4.2 \%)$ patient (Table 1$)$.

Table 1. Proliferating microorganisms (in children).

\begin{tabular}{llllll}
\hline Microorganism name & Man & $\%$ & Woman & $\%$ & Total \\
\hline E. coli & 18 & 66.6 & 9 & 33.3 & $27(75 \%)$ \\
\hline P. aeruginosa & 2 & 100 & 0 & 0 & $2(5.5 \%)$ \\
\hline E. coli+P. aeruginosa & 3 & 60 & 2 & 40 & $5(14 \%)$ \\
\hline Enterococcus faecalis & 1 & 100 & 0 & 0 & $1(2.7 \%)$ \\
\hline Klebsiella oxytoca & 0 & 0 & 1 & 100 & $1(2.7 \%)$ \\
\hline Total & 24 & 66.6 & 12 & 33.3 & $35(100 \%)$ \\
\hline
\end{tabular}

In thirty-two patients with $E$. coli growth, Ampicillinsulbactam (SAM), the most common empiric antibiotics, susceptibility was observed in $13(36.1 \%)$ patients and the number of patients with moderate susceptibility was found as 3 $(8.3 \%)$. Ceftriaxone (CRO) resistance and positivity of broadspectrum beta lactamase (ESBL) were detected in $12(33.3 \%)$ and $10(31.2 \%)$ patients, respectively. Moderate susceptibility to other antibiotics such as Gentamycin (GN), Piperacillin/ Tazobactam were detected in $3(8.3 \%), 3(8.3 \%)$ and $1(2.7 \%)$ patients, respectively. Resistance to cefoperazone-sulbactam and to all cephalosporins was determined in one $(2.7 \%)$ for each sample (Figure 1).

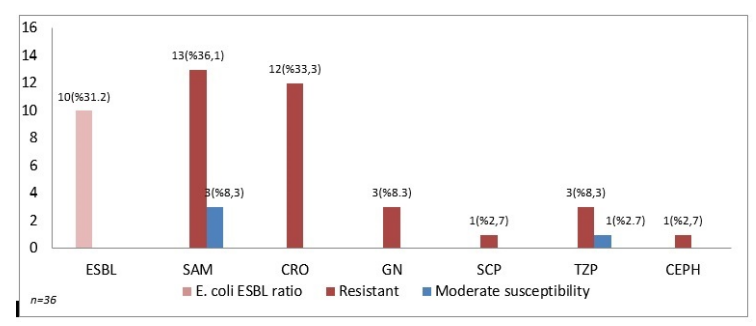

Figure 1. SAM: Ampicillin/Sulbactam, CRO: Ceftriaxone, GN: Gentamycine, SCP: Cefoperazone/Sulbactam, TZP: Piperaciline/ Tazobactam, CEPH: Cephalosporin.

The number of patients having bacterial growth in their peritoneal fluid was 23, and only $E$. coli was detected in 18 (78\%), only Pseudomonas fluorencis was detected in $1(6.2 \%)$, Klebsiella pneumonia+Proteus mirabilis, and E. coli + Pseudomonas aeruginosa in one for each patient, and Citrobacter braaki was grown in one sample (Table 2).

Table 2. Proliferating microorganisms (in adults).

\begin{tabular}{llllll}
\hline Microorganism name & Man & $\%$ & Kadin & $\%$ & Total \\
\hline E. coli & 10 & 55.5 & 8 & 44.5 & $18(78 \%)$ \\
\hline Pseudomonas fluorencis & 1 & 100 & 0 & 0 & $1(4.3 \%)$ \\
\hline
\end{tabular}


The importance of culture-antibiogram and evaluation of the empirical antibiotic treatment in peritonitis due to perforated appendicitis acquired intra-abdominal infection

\begin{tabular}{llllll}
\hline Klebsiella pneumonia & 0 & 0 & 1 & 100 & $1(4.3 \%)$ \\
\hline $\begin{array}{l}\text { Proteus mirabilis } \\
\text { Pseudomonas }\end{array}$ & 1 & 100 & 0 & 0 & $1(4.3 \%)$ \\
\hline $\begin{array}{l}\text { aeruginosa } \\
\text { Citrobacter braaki }\end{array}$ & 1 & 100 & 0 & 0 & $1(4.3 \%)$ \\
\hline Total & 13 & 56.5 & 10 & 43,5 & $23(100 \%)$ \\
\hline
\end{tabular}

In 18 samples with $E$. coli growth, 10 (43.4\%) were resistance to SAM, $1(4.3 \%)$ had moderate susceptibility to SAM, 9 (39.1\%) were resistance to CRO, 6 had positive ESBL, and 5 were resistance to TZP and $1(4.3 \%)$ had moderate susceptibility to TZP. Resistance to GN, ciprofloxacin and SCP were detected in $3(13 \%)$ for each sample. Moreover, resistance to all cephalosporins was observed in $1(4.3 \%)$ sample (Figure 2).

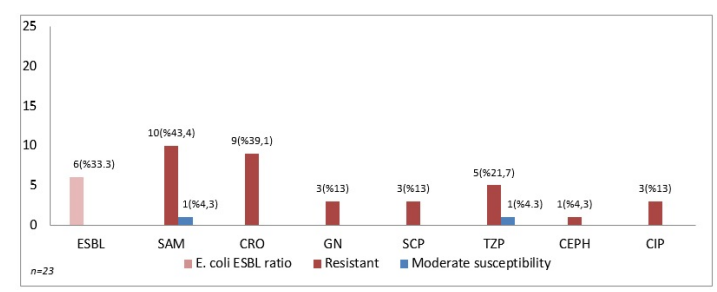

Figure 2. SAM: Ampicillin/Sulbactam, CRO: Ceftriaxone, GN: Gentamycine, SCP: Cefoperazone/Sulbactam, TZP: Piperaciline/ Tazobactam, CEPH: Cephalosporin CIP: Ciprofloxacin.

\section{Discussion}

In most of the studies, severe bacterial contamination and morbidity were reported due to the perforation of appendix, one of the reasons of secondary peritonitis [3-6]. According to the high incidence of gram negative infections in hotter summer months, it was thought that variations in active microorganisms were depending on the seasons [7]. However, dominant pathogens in peritonitis occurring as a result of appendix perforation were shown to be originating from $E$. coli and anaerobic microorganisms (Bacteroides fragilis) [3-7]. In the present study, E. coli growth was detected in 32 of 35 pediatric patients, and 18 of 23 adult patients with peritonitis in line with these studied; however, bacterial growths were not shown variation in a year. Bacteroides fragilis could not be evaluated due to absence of anaerobic culturing.

Obinwa et al. performed a 14 y retrospective study and investigated patients having bacterial growth in their peritoneal cultures of secondary peritonitis following 69 appendicitus in patients at the age group of 1-14 y [8]. As a result of their investigation single and multi-isolate were reported in 31 $(45 \%)$ and $38(55 \%)$ of 69 patients, respectively. E. coli was determined in 56 of 69 isolates $(81 \%)$ and only 4 of them were resistance to amoxicillin-clavulanate acid and 1 was resistance to gentamicin. Moreover, P. aeruginosa was isolated in 4 of 69 patients $(6 \%)$ all of which were sustainable to gentamicin, and Streptococcus spp. were isolated in 5 of 69 patients (7\%) all of which were sustainable to amoxicillin-clavulanate acid. Resistance to Broad Spectrum Beta-Lactamase (ESBL) or

carbapenem was reported in none of the isolates of Enterobacteriaceae growing in peritoneal cultures. In our patients, single isolate was grown in $86.9 \%$ and $85.7 \%$ of adult and pediatric patients, respectively, and only E. coli growth was detected in $78 \%$ and $77 \%$ of these patients, respectively. In growing strains the ratios of ESBL were reported as $33.3 \%$ and $31.2 \%$ in adult and pediatric patients, respectively, and this was attributed to the inappropriate and excess antibiotic consumption.

Castagnola et al. evaluated community acquired peritonitis following appendicitis in cultures of peritoneal fluid on children [9]. A total of 114 strains were examined including 83 strains of $E$. coli, 15 strains of $P$. aeruginosa, 6 strains of enterococcus and 10 strains of other gram negative bacteria, and the ratio of ampicillin/sulbactam and ertapenem resistance were determined as $37 \%$ and $13 \%$, and all of the P. aeruginosa strains were found to be resistance to both antimicrobial agents.

The efficacy of therapy was increased in the ratio between $99 \%$ and $100 \%$ with the combined therapy of gentamicin. While no resistance was observed to meropenem, resistance to piperacillin/tazobactam was reported as $3 \%$. It was stated that combined gentamicin therapy or piperacillin/tazobactam or meropenem single therapy was recommended to those patients instead of single therapies of ampiciline/sulbactam or ertapenem. Dumont et al. evaluated microbiological and antimicrobial susceptibility of isolates growing in peritoneal cultures of pediatric patients who were operated due to community-acquired peritonitis culture in a single surgical center [3]. They investigated a total of 70 patients with peritonitis due to appendicitis $(n=69)$ and small bowel perforation $(\mathrm{n}=1)$. Main pathogens were found as $E$. coli and anaerobic agents in community-acquired peritonitis among children. In the respective study, the resistance ratio of $10 \%$ to amoxicilline-clavulanic acid was emphasized to be independent from post-op risk factors. In the present study, $E$. coli growth was seen in 50 (number of pediatric patients: 32 , number of adults: 18) of 58 patients with peritonitis (number of pediatric patients: 35, number of adults: 23) when the microorganisms were investigated in terms of their species. When it was compared to antibiotic resistance, the resistance to SAM and CRO was found as $13(36.1 \%)$ and $12(33.3 \%)$ in 32 pediatric patients. The resistance to SAM and CRO was found as $10(43.4 \%)$ and $9(39.1 \%)$ in 18 adult patients. Total ESBL positivity was detected as $9(31.2 \%)$ and $6(33.3 \%)$ in pediatric and adult patients, respectively. In most of the studies, it was found to be more common in elderly patients having mental confusion, obesity or immune-specific condition and it was responsible for the high incidence of generalized peritonitis and mortality and the mortality and morbidity rate of appendicitis were reported as 2 to $14 \%$, and $40 \%$ in elderly patients, respectively $[7,10-13]$. In the present study, the age range of adult patients was 18 to $65 \mathrm{y}$. We did not have any patient with immune system disorder and no death was reported due to this disease. 
In worldwide and European countries, resistance to pathogens related to enterobacteriaceae was encountered in currently used antibiotics and even in new generation antibiotics [14]. In the present study, the rate of ESBL was determined as $31.2 \%$ and $33.3 \%$ in pediatric and adult patients, respectively, resistance to all cephalosporin was found in one for each sample $(2.7 \%-4.3 \%)$.

Due to prediction of flora to be able to contaminate the peritoneal surface and use of broad spectrum antimicrobial agents including these organisms initially, it was stated that the requirement of culturing might be reduced [15]. However, certain studies caused the continuation of peritoneal swap application. In the absence of data coming from peritoneal cultures, current condition and variations in infectivity emphasized the inhibition of a predictable guide for empiric antimicrobial therapy in the future $[16,17]$. In various studies, the use of intraoperative peritoneal swap routinely and properly might provide significant and instructive data, and the necessity of peritoneal fluid culturing was stated in the presence of visible inflammation or in high-risk patients [15]. Moawed et al. suggested the use of peritoneal culture for the epidemiological follow-up of rarely isolated microorganisms that are responsible for severe diseases, the benefit of peritoneal swap and a proof for the susceptibility of microbial antibiotic were reported in complex individual patients [16]. In a study conducted by Solomkin et al., it was recommended to sample in perforated appendicitis and other communityacquired intraabdominal infections in case of a resistance problem of frequently detected isolated against common antibiotics (resistance $10 \%$ to $20 \%$ ) in community, and it would be beneficial to detect epidemiological variations and post-discharge therapy choice in case of continuation of routine culturing in low-risk community-acquired intraabdominal infections [18]. In our patients, the rate of resistance to common antibiotics was found above $20 \%$, and routine culturing has been performed in all perforated appendicitis and community-acquired intraabdominal infections.

As it was reported that the spectrum of microbial infection simultaneously varied with antibacterial resistance pattern in terms of geographical differences, and local cultures were beneficial in exposing these variations $[19,20]$. Therefore, we evaluated culture results of patients admitted to the hospital due to perforated appendicitis in our territory. No significant difference was found in terms of resistance in this territory, and it might show variations due to the types of antibiotic usage in surrounding cities.

\section{Conclusion}

Nowadays, the rapid rise in bacterial resistance caused searching alternative solutions for to antibiotic use. The reasons of this include inadequate number of new generation antibiotics and developing bacterial resistance to these antibiotics right from the start. Rational use of antibiotics has been one of the indispensable factors in recent years. Culture antibiograms are generating the most important step of this process. Its significance has been raised in infections requiring empiric antibiotic therapy. In the treatment of secondary peritonitis which is one of the diseases requiring empiric antibiotic therapy, recommended antibiotics have been varied in time. By keeping it current, empiric have gained importance in intraoperative peritoneal culturing and culture-antibiogram results of each region to minimize mortality and morbidity and they should review their own treatment options.

\section{References}

1. Wittmann DH, Schein M, Condon RE. Management of secondary peritonitis. Ann Surg 1996; 224: 10-18.

2. Wittmann DH, Bergstein JM, Frantzides CT. Calculated empiric antimicrobial therapy for mixed surgical infections. Infection 1991; 19: 345-350.

3. Dumont R, Cinotti R, Lejus C, Caillon J, Boutoille D, Roquilly A. The microbiology of community-acquired peritonitis in children. Pediatr Infect Dis J 2011; 30: 131-135.

4. Brook I. Bacterial studies of peritoneal cavity and postoperative surgical wound drainage following perforated appendix in children. Ann Surg 1980; 192: 208-212.

5. Lin WJ, Lo WT, Chu CC, Chu ML, Wang CC. Bacteriology and antibiotic susceptibility of communityacquired intra-abdominal infection in children. J Microbiol Immunol Infect 2006; 39: 249-254.

6. Bennion RS, Baron EJ, Thompson JE Jr, Downes J, Summanen P, Talan DA. The bacteriology of gangrenous and perforated appendicitis-revisited. Ann Surg 1990; 211: 165-171.

7. Perencevich EN, McGregor JC, Shardell M, Furuno JP, Harris AD, Morris Jr JG. Summer peaks in the incidences of gram-negative bacterial infection among Hospitalized patients. Infect Control Hosp Epidemiol 2008; 29: 1124-1131.

8. Obinwa O, Casidy M, Flynn J, The microbiology of bacterial peritonitis due to appendicitis in children. Ir J Med Sci 2014; 183: 585-591.

9. Castagnola E, Bandettini R, Ginocchio F, Perotti M, Masa DL, Ciucci A. Susceptibility to antibiotics of aerobic bacteria isolated from community acquired secondary peritonitis in children: therapeutic guidelines might not always fit with and everyday experience. J Chemother 2013; 25: 213-216.

10. Kraemer M, Franke C, Ohmann C, Yang Q. Actute appendicitis in late adulthood: incidence, presentation, and outcome. Results of a prospective multicenter acute abdominal pain study and a review of the literature. Arch Surg 2000; 385: 470-481.

11. Blanc B, Pocard M. Surgical techniques of appendectomy for acute appendicitis. J Chir 2009; 146: 22-31.

12. Terasawa T, Blackmore CC, Bent S, Kohlwes RJ. Systematic review: computed tomography and ultrasonography to detect acute appendicitis in adults and adolescents. Ann Intern Med 2004; 141: 537-546. 
The importance of culture-antibiogram and evaluation of the empirical antibiotic treatment in peritonitis due to perforated appendicitis acquired intra-abdominal infection

13. Sanda RB, Seliem SI, Omar E, Ashraf S. Perforated appendicitis in a septuagenarian. Ann Afr Med 2011; 10: 249-251.

14. Rabasseda X. A report from the 20th European Congress of clinical microbiology and infectious diseases 2010; 46: 533-542.

15. Davies Huw OB, Alkhamesi NA, Dawson P.M. Peritoneal fluid culture in appendicitis: Review in changing times. Int J Surg 2010; 8: 426-429.

16. Moawad MR, Dasmohapatra S, Justin T, Keeling N. Value of intraoperative abdominal cavity culture in appendicectomy: a retrospective study. Int $\mathrm{J}$ Clin Pract 2006; 60: 1588-1590.

17. Gladman MA, Knowles CH, Gladman LJ, Payne JG. Intraoperative culture in appendicitis: traditional practice challenged. Ann R Coll Surg Engl 2004; 86: 196-201.

18. Solomkin JS, Mazuski JE, Bradley JS, Rodvold KE, Goldstein Ellie JC, Baron Ellen J. Diagnosis and management of complicated intra-abdominal infection in adults and children: Guidelines by the surgical infection society and the infectious diseases society of America. Clin Infect Dis 2010; 50: 133-164.
19. Sonnenberg A. Seasonal variation of enteric infections and inflammatory bowel disease. Inflamm Bowel Dis 2008; 14: 955-959.

20. McCormick AW, Whitney CG, Farley MM, Lynfield R, Harrison LH, Bennett NM. Geographic diversity and temporal trends of antimicrobial resistance in Streptococcus pneumoniae in the United States. Nat Med 2003; 9: 424-430.

\section{*Correspondence to}

Hakan Sezgin Sayiner

Faculty of Medicine

Department of Clinical Infectious Diseases and Medical Microbiology

Adiyaman University

Training and Research Hospital

Adiyaman

Turkey 